\title{
Highly Ionized Ar Plasma Waveguides Generated by a Fast Capillary Discharge
}

\author{
B. M. Luther, Y. Wang, M. Berrill, D. Alessi, M. C. Marconi, M. A. Larotonda, and J. J. Rocca, Fellow, IEEE
}

\begin{abstract}
Highly ionized Ar plasma channels were created by a fast capillary discharge and used to guide laser pulses with peak intensities up to $2.2 \times 10^{17} \mathrm{~W} / \mathrm{cm}^{2}$ over a 5.5 -cm distance. These plasmas are of interest for the generation of efficient soft $\mathrm{X}$-ray lasers by longitudinal laser excitation. The guides were characterized using plasma interferometry, modeling, and near field imaging.
\end{abstract}

$\mathbf{T}$ HE guiding of intense laser beams in plasmas has attracted significant attention [1]-[7]. This is motivated by the need of extending the interaction length between intense laser pulses and plasmas beyond the limitations set by diffractive defocusing and ionization-induced refraction. An application of considerable interest for plasma waveguides is the longitudinal excitation of soft X-ray lasers that can potentially result in saturated amplifiers with reduced laser pump energy and increased efficiency.

Herein, we report images related to the characterization of multiply ionized plasma waveguides created by a fast Ar capillary discharge of the type used to develop collisional soft X-ray lasers [8]. The experiments used $\sim 2.5 \mathrm{ps,} 800$-nm Ti:Sa laser pulses, except for the demonstration of high-intensity beam guiding that was done using $\sim 100$ fs pulses. The discharge utilized 3.2-mm diameter, 5.5-11-cm-long $\mathrm{Al}_{2} \mathrm{O}_{3}$ capillaries filled with 180 to 150 mtorr of Ar. The discharge current pulses had a peak amplitude of 15-21 kA, and a half-period of 120 ns. Interferometry of the plasma shows the evolution of an annular plasma shell with density minimum at the center that constitutes a waveguide of continuously decreasing diameter and increasing density until it collapses on axis (Fig. 1). The plasma compression is accompanied by an increase in the degree of ionization, reaching $\mathrm{Z}=8$ shortly before the collapse, as corroborated during these experiments by the observation of lasing at $46.9 \mathrm{~nm}$ in Ne-like Ar.

The guiding properties of a 5.5 -cm-long capillaries were investigated by imaging the output of the capillary [Fig. 2(right)] and by

\footnotetext{
Manuscript received July 2, 2004; revised November 4, 2004. This work was supported in part by the National Science Foundation under Grant ECS9977677, in part by the National Science Foundation Engineering Research Center for Extreme Ultraviolet Science and Technology, in part by the W.M. Keck Foundation, and in part by the U.S. Department of Energy, Chemical Sciences, Geosciences and Biosciences Division of the Office of Basic Energy Science.

The authors are with the National Science Foundation Engineering Research Center for Extreme Ultraviolet Science and Technology and they are also with Electrical and Computer Engineering Department, Colorado State University, Engineering Research Center, Fort Collins, CO 80523 USA (e-mail: bml@lamar.colostate.edu).
}

Digital Object Identifier 10.1109/TPS.2005.845267 modeling the beam propagation using the Kirchhoff-Fresnel integral inputing the radially dependent phase delay determined from the interferometrically measured density profile [Fig. 2(left)]. For times less than $30 \mathrm{~ns}$, with respect to beginning of the current pulse, the plasma density is low and the peak of the electron density is far from the axis, resulting in no guiding of the beam. When the plasma column compresses to $\sim 800 \mu$ min diameter, the wings of the laser pulse begin to experience a phase shift that results in output modes with concentric circles as shown in Fig. 2(a). As the column continues to compress a variety of lower order modes, such as that illustrated in Fig. 2(b), are seen. Fig. 2(c) shows an approximately gaussian output mode with a full-width half-maximum (FWHM) of $\sim 50 \mu \mathrm{m}$. Subsequently, the guide is lost as the plasma pinches and the electron density profile becomes convex, resulting in the beam being strongly refracted, as shown in Fig. 2(d). The development of a second guiding phase was observed to take place during the first few nanoseconds of the expansion that follows the pinch, as seen in Fig. 2(e). However, the post-pinch guides are significantly more leaky.

Channels composed of Ar VIII or Ar IX ions are immune to tunnel ionization for intensities $<3 \times 10^{16} \mathrm{Wcm}^{-2}$ and $<1.5 \times 10^{18} \mathrm{Wcm}^{-2}$, respectively. We verified that the prepinch matched guides are maintained at high intensities by injecting pulses with a measured average energy of $500 \mathrm{~mJ}$ and pulse duration of $112 \mathrm{fs}$, corresponding to a peak input intensity of $2.2 \times 10^{17} \mathrm{Wcm}^{-2}$. Full details are given in [6].

\section{REFERENCES}

[1] C. G. Durfee and H. M. Milchberg, "Light pipe for high-intensity laserpulses," Phys. Rev. Lett., vol. 71, pp. 2409-2412, 1993.

[2] Y. Ehrlich et al., "Guiding of high intensity laser pulses in straight and curved plasma channel experiments," Phys. Rev. Lett., vol. 77, pp. 4186-4189, 1996.

[3] A. Butler et al., "Guiding of high-intensity laser pulses with a hydrogen-filled capillary discharge waveguide," Phys. Rev. Lett., vol. 89, p. $185003,2002$.

[4] T. Hosokai et al., "Optical guidance of terrawatt laser pulses by the implosion phase of a fast Z-pinch discharge in a gas-filled capillary," Opt. Lett., vol. 25, pp. 10-12, 2000.

[5] D. Kaganovich et al., "High efficiency guiding of terawatt subpicosecond laser pulses in a capillary discharge plasma channel," Phys. Rev. E., Stat. Phys. Plasmas Fluids Relat. Interdiscip. Top., vol. 59, pp. R4769-R4772, 1999.

[6] B. M. Luther et al., "Guiding of intense laser beams in highly ionized plasma columns generated by a fast capillary discharge," Phys. Rev. Lett., vol. 92, p. $235002,2004$.

[7] C. G. R. Geddes et al., "High-quality electron beams from a laser wakefield accelerator using plasma-channel guiding," Nature, vol. 431, pp. 538-541, 2004

[8] B. R. Benware, C. D. Macchietto, C. H. Moreno, and J. J. Rocca, "Demonstration of a high average power tabletop soft X-ray laser," Phys. Rev. Lett., vol. 81, pp. 5804-5807, 1998. 

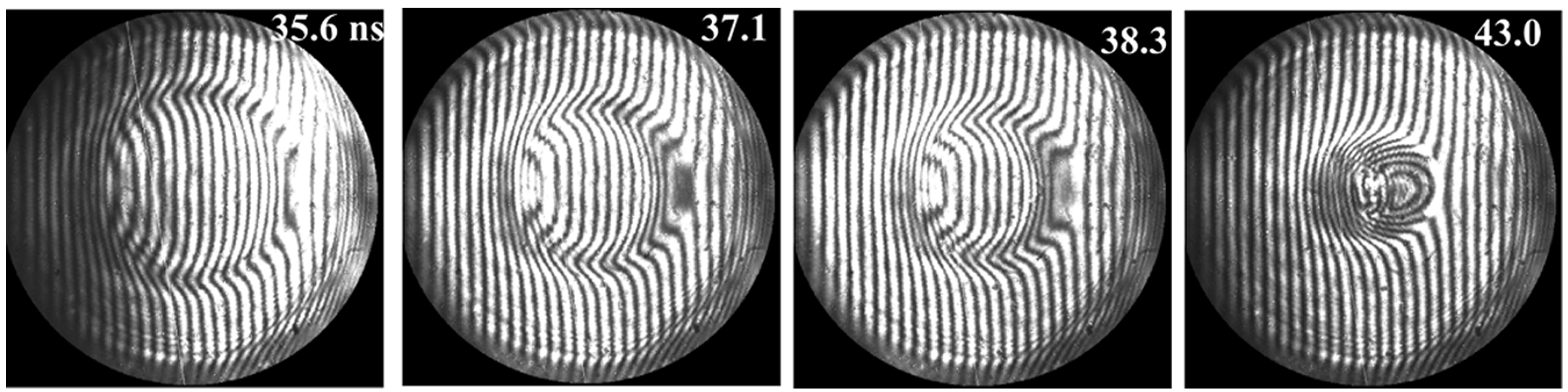

Fig. 1. Time progression of interferograms showing the plasma column compression and waveguide development for a $250 \mathrm{mtorr}$ Ar discharge in a 3.2 -mm diameter, 11-cm-long capillary. Times are measured with respect to the current pulse.
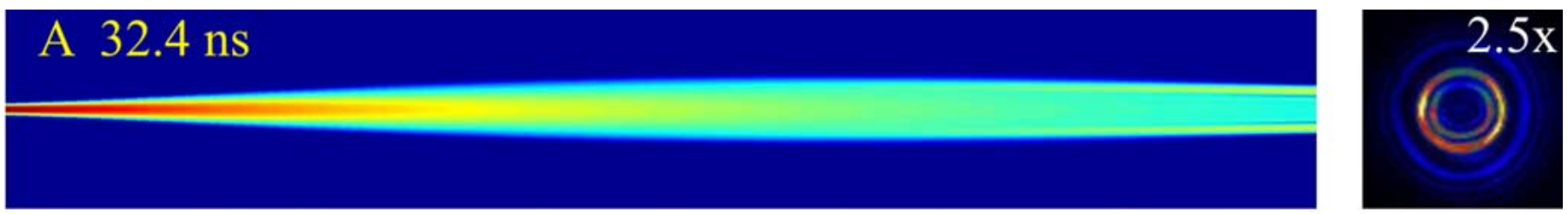

\section{B $33.2 \mathrm{~ns}$}

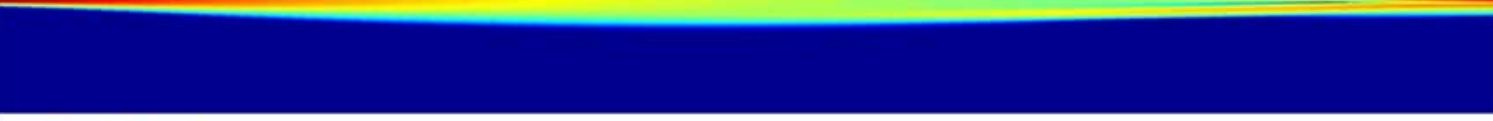

\section{33.6 ns}
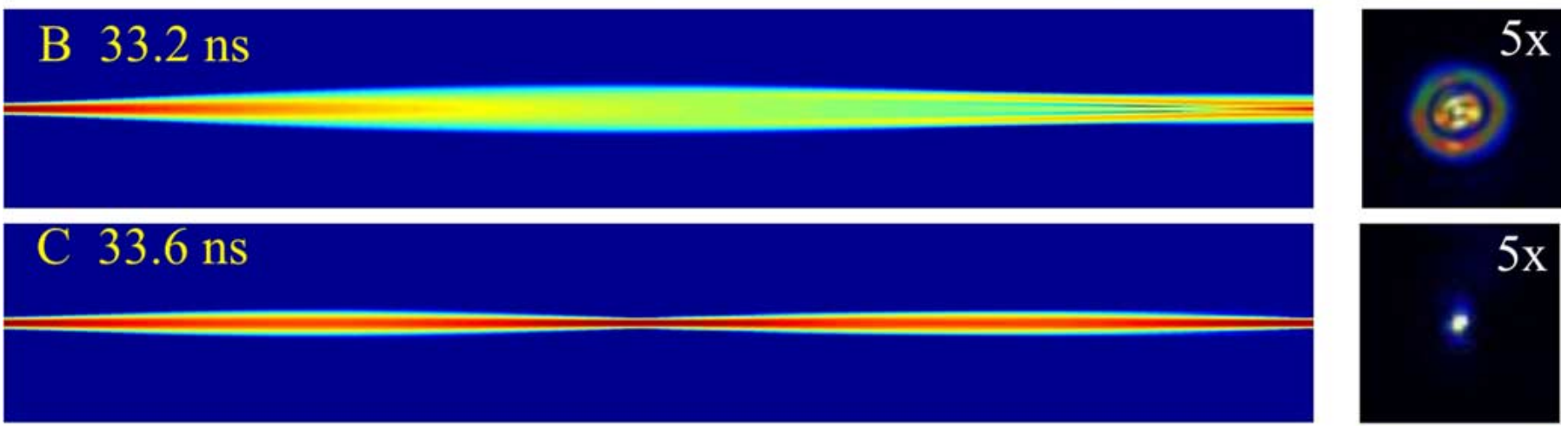

\section{D $34.8 \mathrm{~ns}$}

\section{E 36.8 ns}

Fig. 2. Modeled cross sections showing the calculated guided beam propagation in a 5.5-cm capillary (left), and corresponding experimental exit mode images (right, with magnification given). 